\title{
Beam Calorimetry at the Large Negative Ion source Test Facility ELISE: Experimental Setup and Latest Results
}

\author{
Riccardo Nocentini ${ }^{\mathrm{a}}$, Federica Bonomo ${ }^{\mathrm{a}}$, Marina Ricci ${ }^{\mathrm{a}}$, Antonio Pimazzoni ${ }^{\mathrm{b}, \mathrm{c}}$, Ursel Fantz ${ }^{\mathrm{a}}$, Bernd \\ Heinemann $^{\mathrm{a}}$, Rudolf Riedl ${ }^{\mathrm{a}}$, Dirk Wünderlich ${ }^{\mathrm{a}}$. \\ ${ }^{a}$ Max-Planck-Institut für Plasmaphysik, Boltzmannstr. 2, Garching, Germany \\ ${ }^{b}$ Consorzio RFX, Corso Stati Uniti 4, 35127 Padova, Italy \\ ${ }^{c}$ Università degli studi di Padova, Via 8 Febbraio 2, 35122 Padova Italy
}

\begin{abstract}
The test facility ELISE is the first step within the European roadmap for the development of the ITER NBI system. ELISE is equipped with a $1 \times 0.9 \mathrm{~m}^{2}$ Radio Frequency negative ion source (half the ITER source size) and an ITER-like 3-grid extraction system which can extract an $\mathrm{H}^{-}$or $\mathrm{D}^{-}$beam for $10 \mathrm{~s}$ every 3 minutes (limited by available power supplies) with a total acceleration voltage of up to $60 \mathrm{kV}$. In the beam line of ELISE several beam diagnostic tools have been installed with the aim to evaluate beam intensity, divergence and uniformity.

A copper diagnostic calorimeter gives the possibility to measure the beam power density profile with high resolution. The measurements are performed by an IR micro-bolometer camera and 48 thermocouples embedded in the calorimeter. A gaussian fit procedure has been implemented in order to characterize the large negative ion beam produced by ELISE.

The latest results obtained from the beam calorimetry at ELISE show that the average beamlet group inhomogeneity is maximum $13 \%$. The measured beam divergence agrees with the one measured by beam emission spectroscopy within $30 \%$.
\end{abstract}

Keywords: ITER, Neutral Beam Injection, Negative Ion Source, RF source, Beam diagnostics, Calorimeter.

\section{Introduction}

A neutral beam injection (NBI) system based on negative hydrogen ions is currently under development to provide additional heating power and current drive for the international nuclear fusion experiment ITER [1,2]. In the past decades the Max-Planck-Institut für Plasmaphysik (IPP) in Garching, Germany, has developed negative ion radio frequency (RF) sources on which the ITER NBI sources are based [3-7]. The IPP RF prototype sources have achieved the ITER requirements, but not simultaneously and with an extraction area smaller (up to $200 \mathrm{~cm}^{2}$ ) than what is required for ITER $\left(2000 \mathrm{~cm}^{2}\right)$. Therefore recently the testbed ELISE [8-10] was built, as an intermediate step between the small prototype sources and the ITER sources, in the framework of the Fusion for Energy (F4E) R\&D roadmap for the ITER NBI system. Its goal is to reach ITER-relevant parameters [11] simultaneously and to demonstrate plasma and beam uniformity in an ITER-sized negative ion source.

ELISE has an extraction area of $1000 \mathrm{~cm}^{2}$ and a source area of about $1 \times 0.9 \mathrm{~m}^{2}$, which is the same width, but half the ITER source height. The ion source can produce an $\mathrm{H}$ or $\mathrm{D}$ plasma for up to $1 \mathrm{~h}$ and accelerate a negative ion beam up to $60 \mathrm{kV}$ for $10 \mathrm{~s}$ every $3 \mathrm{~min}$. The three-grid extraction system of ELISE is ITER-like, with a total of 640 apertures arranged in 2 horizontal rows (beam segments) of 4 beamlet groups (BGs) each $[8,12]$. Inside the source, in front of the extraction area, a magnetic filter field (FF) of up to $5 \mathrm{mT}$ can be produced by means of an electric current of up to 5.23 kA that flows through the first grid and properly placed return conductors [13].

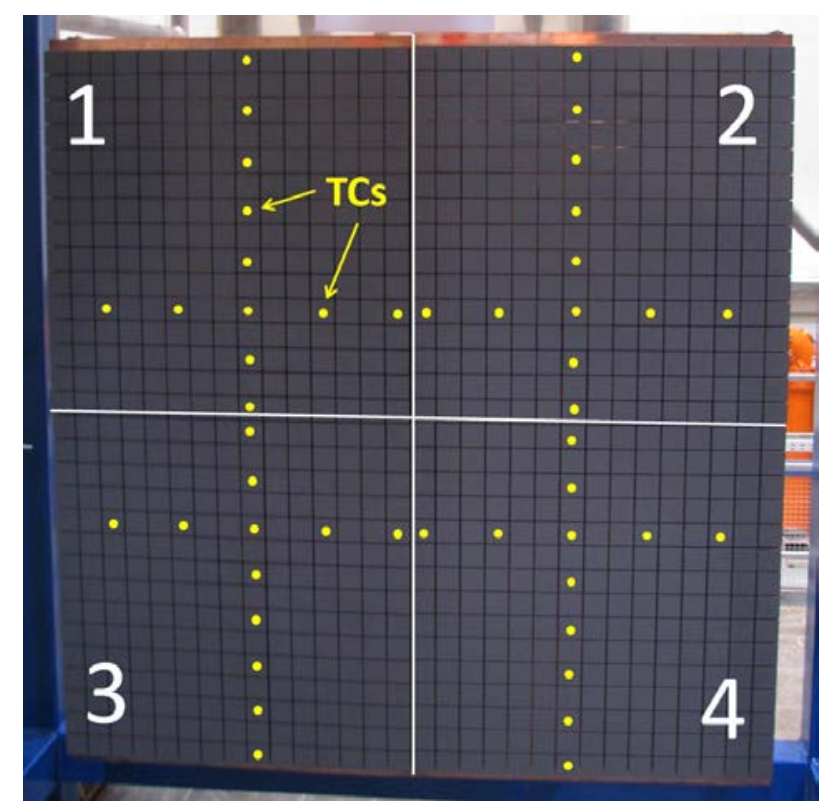

Fig. 1. Diagnostic calorimeter of ELISE. The four quadrants corresponding to the four copper plates are shown, as well as the $\mathrm{MoS}_{2}$ black coating and the positions of the 48 embedded thermocouples (TCs).

Operation of ELISE started in the beginning of 2013. The results of the experimental campaigns with Cs and short plasma pulses have been summarized in [10]. 
Several diagnostic tools are employed to evaluate plasma [14] and beam [15] properties. This paper summarizes the experimental setup, evaluation methods and the latest results of the beam calorimetry in ELISE.

\section{The diagnostic calorimeter}

A sophisticated copper diagnostic calorimeter has been designed and manufactured at IPP with the aim to obtain information about the beam of ELISE. An infrared (IR) camera looks at the calorimeter to measure the superficial 2D temperature distribution, which is used to obtain the beam power distribution. To obtain a sufficiently precise measurement the calorimeter is covered on the beam-facing side with a black $\mathrm{MoS}_{2}$ spray coating with high emission coefficient in the IR range. This coating has been tested extensively using a temporary beam dump installed in ELISE during the first experimental phase.

The diagnostic calorimeter, shown in figure 1 , is made of 4 copper plates with a size of $600 \mathrm{~mm}$ x $600 \mathrm{~mm}$, which are installed at $3.5 \mathrm{~m}$ from the extraction system. A piping structure supports the copper plates and provides the cooling water. Separated water outlets and inlets allow for a beam power measurement based on water calorimetry for each one of the four calorimeter quadrants.

Each calorimeter plate is made of a copper back plate, with embedded cooling water circuit, and 15 x 15 copper blocks (beam facing area: $38 \mathrm{~mm}$ x $38 \mathrm{~mm}$, thickness: $25 \mathrm{~mm}$, gap between blocks: $2 \mathrm{~mm}$ ) which are individually brazed on the beam-facing side of the back plate with an intermediate thermal resistance. Each block operates therefore as a small inertial calorimeter that stores the heat during the beam pulse and slowly cools down in the pause between beam pulses. The blocks and thermal resistances are designed so that the power deposited in a block during the beam-on phase is approximately proportional to the temperature increase of the block, allowing for easy calculation of the average beam power impinging a block during the pulse. 48 thermocouples (TCs) have been embedded in the calorimeter to calibrate the IR thermography, and to provide a beam profile measurement over two vertical and two horizontal lines independently from the IR thermography.

\section{IR measurements}

A micro-bolometer IR camera observes the diagnostic calorimeter, with the aim to measure the average temperatures of each block. Through an evaluation procedure it is possible to obtain a $2 \mathrm{D}$ image of the beam power distribution with a total resolution of $30 \times 30$ "pixels". The IR camera has a spectral range of 7.8$14 \mu \mathrm{m}$ and a resolution of 640 (h) $x 480$ (v) pixels. The camera is equipped with a wide angle objective that provides full view of the calorimeter. An image of the calorimeter seen from the IR camera right before the end of shot \#10761 is shown in figure 2. The beam heats up the central part of the calorimeter and the top and bottom beam segments can be recognized due to the relatively low beam divergence $\left(1^{\circ}\right)$. The beam is deflected downwards due to the magnetic FF.

\subsection{IR calibration}

In order to perform a precise temperature measurement by means of the IR camera, the emissivity coefficient of the black surface has to be known with sufficient accuracy. During the beam pulse it is not possible to measure this emissivity coefficient. The contact resistance between black coating and copper substrate and the presence of some dust on the coating cause a significant temperature difference between block surface temperature and TC temperature under beam power load. The emission coefficients for the 48 blocks with embedded TCs is estimated by comparing the temperatures measured by TC and the IR signal during the cooling down phase, in between two beam pulses.

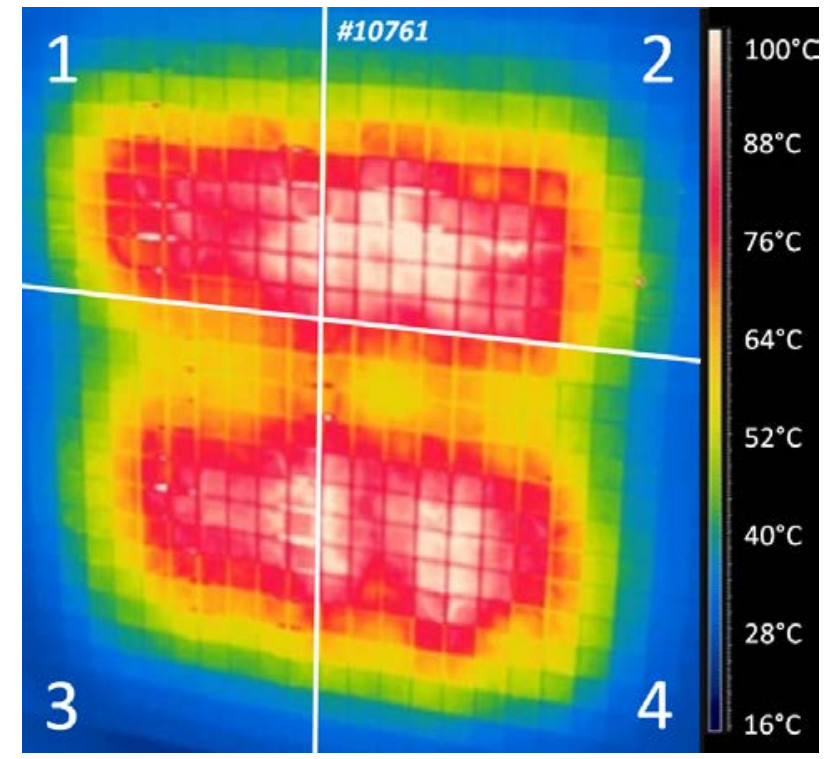

Fig. 2. IR image of the calorimeter for beam pulse \#10761. Pulse parameters: $\mathrm{RF}=2 \mathrm{x} 62 \mathrm{~kW}, \mathrm{U}_{\mathrm{HV}}=30 \mathrm{kV}, \mathrm{I}_{\mathrm{PG}}=2.7 \mathrm{kA}$, $\mathrm{J}_{\mathrm{H}^{-}}=10.5 \mathrm{~mA} / \mathrm{cm}^{2}$, div. $=1^{\circ}$, beam pulse length $=9.5 \mathrm{~s}$. The peak temperature of plate 2 and 4 is slightly higher due to beam non-homogeneity.

In this study it is assumed that the emission coefficient is same for all the 900 blocks of the diagnostic calorimeter and is a constant value. The power density profile of a beam pulse obtained by IR measurements and the corresponding gaussian fit are shown in figure 3.

\subsection{Gaussian fit procedure}

The divergence of the single beamlets that compose the total beam cannot be measured directly. Even for beams with a low divergence (about $1^{\circ}$ ), the beamlets are overlapping at the calorimeter, $3.5 \mathrm{~m}$ downstream of the extraction system. The beam divergence and uniformity are therefore evaluated for each BG through a gaussian fit. In this procedure, 8 gaussian profiles are used to fit as much "sub-beams", corresponding to the BGs.

The number of free parameters needed to perform the gaussian fit is kept small by making a few assumptions: 
the vertical position of the BG centers is equal for the BGs belonging to the same beam segment; the width of the gaussian function (half 1/e), both in the horizontal and the vertical direction are the same for BGs belonging to the same beam segments; in addition, the horizontal position of each beamlet group is assumed to correspond to the geometrical projection of the beamlet groups from the extraction system onto the calorimeter. In this way the total number of free parameters is limited to 14 .

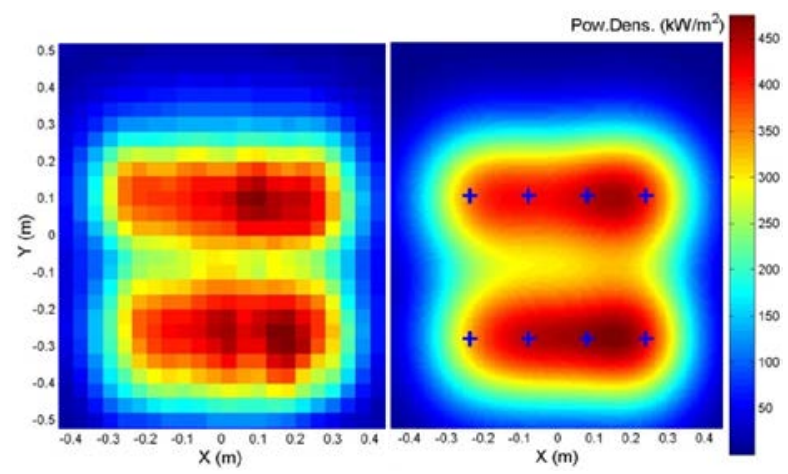

Fig. 3. Left: beam power distribution on the calorimeter of ELISE obtained by IR imaging for beam pulse \#10761. Right: gaussian fit of the data obtained by IR imaging of the calorimeter for the same beam pulse. From this gaussian fit it is possible to characterize the beam divergence and uniformity in terms of intensity and width of the gaussian fit for each beamlet group (centers of BGs are indicated by blue crosses).

Once the gaussian fit has been performed, the widths of the BGs can be used to estimate the beamlet divergence, as discussed in section 4.2, while the amplitudes can be used to estimate the beam uniformity. The procedure has the capability to give relatively accurate information on the beamlet group properties even when the beamlet divergence is larger than the design value $\left(1^{\circ}-2^{\circ}\right)$.

\subsection{Coating degradation}

After several months of operation, “colder” local areas appeared in the calorimeter, which were not visible at the beginning of the experimental campaign (see figure 4). These areas affect the IR measurement, which is performed during the beam off phase.

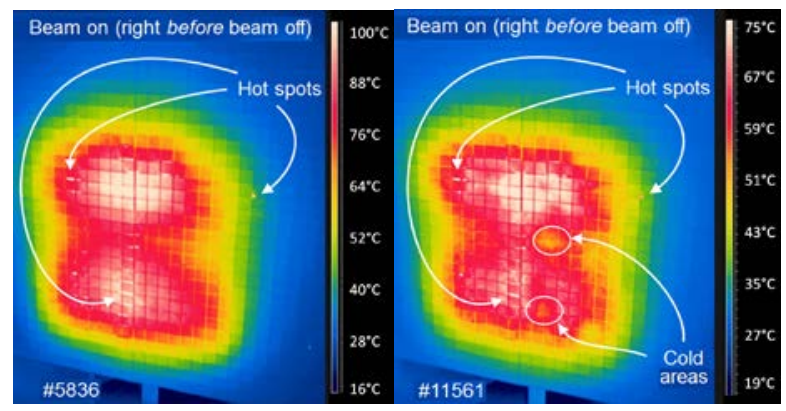

Fig. 4. IR pictures of diagnostic calorimeter during the beam-on phase at the beginning of operation (left) and right before the planned maintenance period (right). "Hot spots" are present in both images and represent areas with thicker coating or weaker adhesion of the coating with the substrate, which are not critical. In the right picture "cold areas" are visible, which correspond to coating degradation or removal.

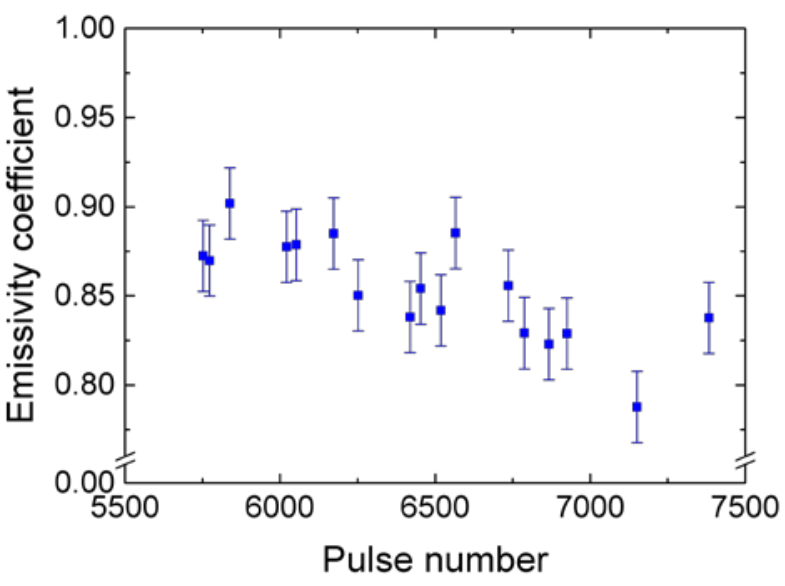

Fig. 5. Average emissivity coefficient of diagnostic calorimeter coating against the pulse number [16]. The average emissivity coefficient shows a decreasing trend with increasing beam operation time.

It has been measured that the average emissivity coefficient slightly decreased with increasing pulse number, as shown in figure 5, suggesting a degradation of the coating due to beam sputtering. In order to simplify the evaluation of the IR images an average emissivity coefficient value $\varepsilon=0.83$ has been chosen. The coating degradation is more significant where the ion bombardment from the beam is higher, i.e. in the areas corresponding to the projection of the BGs, taking into consideration a small downwards beam deflection due to the magnetic FF, as shown in Figure 6.

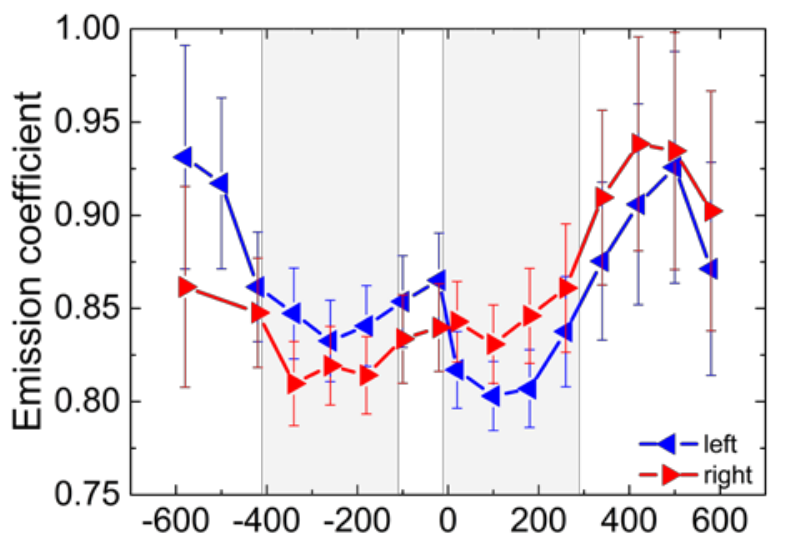

Fig. 6. Distribution of emissivity coefficient for the two (left and right) vertical rows of blocks equipped with TCs [16]. The shaded area corresponds to the position of the beamlet groups, which is shifted downwards by the magnetic filter field.

This degradation became obvious during a maintenance period, when the coated surface of the diagnostic calorimeter could be observed directly. The coating had withstood a total of about 6000 beam pulses (each $10 \mathrm{~s}$ ) up to that point. For the next experimental campaign, the beam-facing surface of the diagnostic calorimeter has been cleaned and sprayed again with $\mathrm{MoS}_{2}$ to restore the initial coating characteristics. 


\section{Evaluation of beam properties}

\subsection{Beam power}

The total beam power for each calorimeter plate can be evaluated by means of water calorimetry as well as by means of IR thermography. Due to a $2 \mathrm{~mm}$ gap between the blocks, the total beam power collected by the sum of the blocks and seen by the IR thermography is lower than the one collected by the whole plate and detected by the water calorimetry. The ratio between the beamfacing surfaces of the blocks and of the whole copper plate is 0.9. Due to the beam divergence a small portion of the beam impacts on the sides of the blocks, contributing for a small additional block temperature increase. The ratio between the two power estimates in therefore slightly higher than 0.9 and this is confirmed by measurements within 5\% of accuracy.

\subsection{Beam divergence and comparison with BES}

Simulations have been performed to find the relation between the divergence of a single beamlet and the width of the beamlet groups obtained by gaussian fit and IR thermography. The simulations reproduce the beam power distribution by superimposing the power distributions (with gaussian profiles) of the 640 beamlets of the extraction system. The divergence and amplitude has been defined equal for every beamlet. The fit procedure has then been applied to the resulting 2D power distribution map on the calorimeter. By changing the value of beamlet divergence a correlation with the beamlet group widths, shown in figure 7 , could be determined.

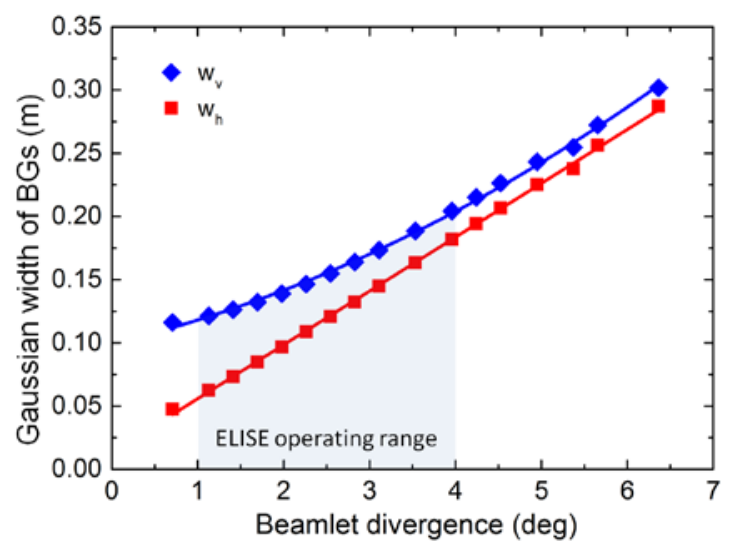

Fig. 7. Result from numerical simulation that links the gaussian width of beamlet groups and the beamlet divergence. The diagram is obtained for the vertical and horizontal gaussian widths, $\mathrm{w}_{\mathrm{v}}$ and $\mathrm{w}_{\mathrm{h}}$, respectively.

The beam divergence evaluation obtained from IR thermography and gaussian fit procedure has been validated against the measurements performed by beam emission spectroscopy (BES).

The BES diagnostic tool is based on the detection of $\mathrm{H}_{\alpha}$ light produced by collisions between the beam ions and residual background gas in the tank. The evaluation of the corresponding spectrum gives an estimate of the beam divergence and stripping losses. At present 20 lines of sight (LoS) are installed, $4 \mathrm{LoS}$ for the horizontal and 16 LoS for the vertical beam profile, as shown in figure 8.

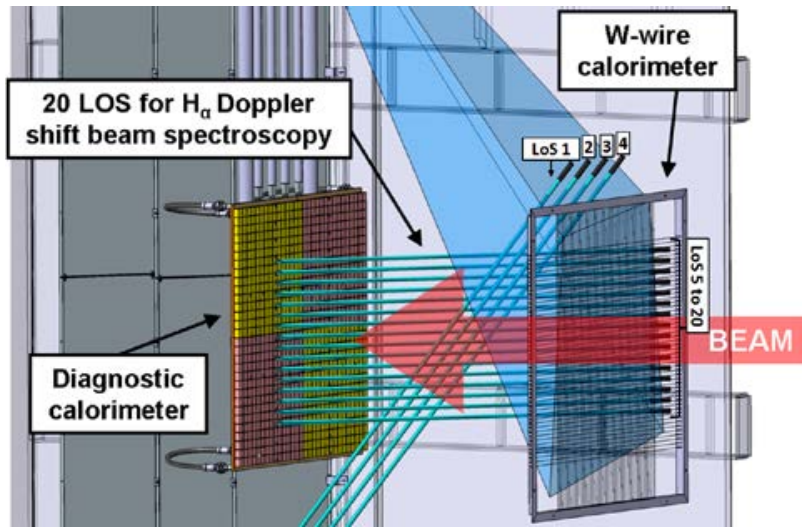

Fig. 8. CAD drawing of the beam diagnostic tools in ELISE. The position and numbering of the 20 LoS for BES is indicated.

The value estimated by BES results $20-30 \%$ smaller than the divergence evaluated by IR thermography. This discordance is being investigated [17].

\subsection{Beam uniformity and beam deflection}

The beam inhomogeneity in ELISE has been measured by comparing the gaussian amplitudes $A_{i}$ to the average row amplitudes $\langle\mathrm{A}\rangle_{\text {top }}$ or $\langle\mathrm{A}\rangle_{\text {bottom. }} \mathrm{A}$ high beam uniformity was found for short pulses at low beam power (10 s HV-phase in a $20 \mathrm{~s}$ RF pulse; RF power up to $2 \times 40 \mathrm{~kW}, \mathrm{U}_{\mathrm{HV}}$ up to $35 \mathrm{kV}$ ). In average the inhomogeneity is smaller in D operation (1\%) than in $\mathrm{H}$ (5\%). The first operational campaign has also shown in $\mathrm{H}$ a higher top-bottom inhomogeneity between the beam segments (up to $30 \%$ ), which is probably due to the source conditioning phases.

During the experimental campaigns with Cs and short plasma pulses, a maximum average inhomogeneity of $13 \%$ for the upper and $9 \%$ for the lower beam segment has been obtained in $\mathrm{H}$ operation, while in $\mathrm{D}$ operation the maximum average inhomogeneity is $11 \%$ for the upper and $6 \%$ for the lower beam segment. It was also observed, as shown in figure 9 , that the beam uniformity increases for lower beam divergence.

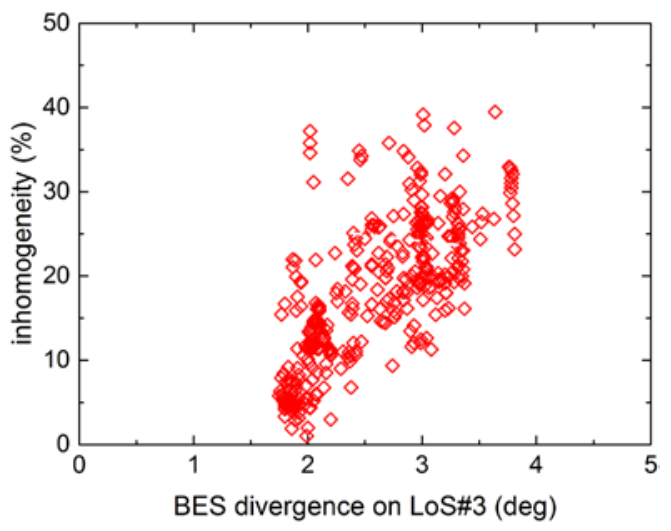

Fig. 9. Beam inhomogeneity (hydrogen operation) within the upper row (from gaussian fit of BGs) against beam divergence, evaluated by means of BES on the vertical LoS\#3. 


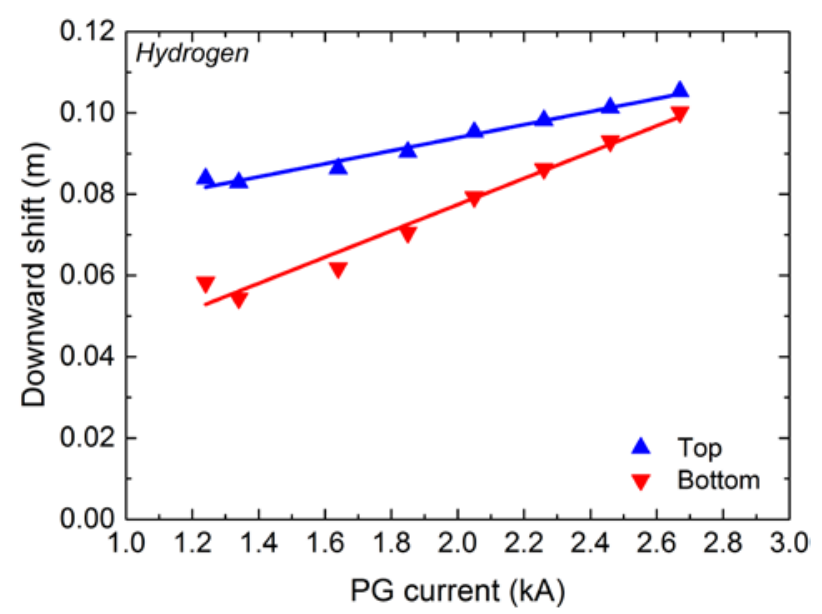

Fig. 10. Downward shift of top and bottom beam segments as a function of the FF (here in terms of PG current, which is proportional to the FF strength).

Also, in a dedicated FF scan, visible in figure 10, different shifts for upper and lower beam segments have been observed. Unexpectedly, for an increase of the FF strength, the bottom beam half shifts downwards more than the upper beam half. These topics are still under investigation.

\section{Conclusions}

The large negative ion source testbed ELISE is equipped with a sophisticated diagnostic calorimeter to evaluate beam characteristics such as beam divergence and uniformity. The calorimeter hosts sensors for water calorimetry and 48 thermocouples which are used to measure beam profiles and to calibrate an IR imaging of the surface. The IR thermography allows for measuring the $2 \mathrm{D}$ beam power distribution on the calorimeter. To increase surface emissivity and measurement accuracy the copper plates of the calorimeter have been coated with a $\mathrm{MoS}_{2}$ black coating. The coating has shown a slight degradation after about 6000 beam pulses, therefore the calorimeter has been coated again during a maintenance phase.

The data obtained by IR thermography has been evaluated by a gaussian fit procedure. This provides the divergence and intensity of the 8 beamlet groups that form the whole beam. A numerical simulation has been performed to obtain the beamlet divergence from the BG gaussian widths. The beam divergence measured by IR thermography in the last year of operation at ELISE varied between $1^{\circ}$ and $4^{\circ}$. These results differ about 20 $30 \%$ compared with the values measured by beam emission spectroscopy. The beam inhomogeneity has been measured in terms of difference between the intensities of the beamlet groups obtained by the gaussian fit. The average BG inhomogeneity was maximum $13 \%$ and was found to decrease for lower beam divergence. In addition, increasing the FF strength, the bottom beam half is deflected downwards more than the upper beam half. These results are currently under investigation.

\section{References}

[1] R. Hemsworth, et al., "Status of the ITER heating neutral beam system", Nuclear Fusion, v49, 045006, (2009).

[2] B. Schunke, et al., "Status of the negative ion based Diagnostic Neutral Beam for ITER," Proceedings of the 24th IAEA Fusion Energy Conference. (2012) p. ITR/P103.

[3] E. Speth, et al., "Overview of the RF source development programme at IPP Garching," Nucl. fusion 46 (2006) 220.

[4] A. Stäbler, et al. "Development of a RF-driven ion source for the ITER NBI system," Fus. Eng. Des. 84, 265-268 (2009).

[5] W. Kraus, et al., "The development of the radio frequency driven negative ion source for neutral beam injectors," Review of Scientific Instruments 83 (2012) $02 \mathrm{~B} 104$.

[6] P. Franzen, et al., "Progress of the development of the IPP RF negative ion source for the ITER neutral beam system," Nucl. Fusion 47 (2007) 264.

[7] U. Fantz, et al., "Physical performance analysis and progress of the development of the negative ion RF source for the ITER NBI system," Nucl. Fusion 49 (2009) 125007.

[8] B. Heinemann, et al., "Design of the "half-size" ITER neutral beam source for the test facility ELISE," Fusion Eng. Des. 84 (2009) 915.

[9] B. Heinemann, et al., "The negative ion source test facility ELISE," Fusion Eng. Des. 86 (2011) 768.

[10] P. Franzen, et al., "Status of the ELISE test Facility," AIP conference proceedings 1655, (2015).

[11] R. Nocentini, et al., "Towards a large RF ion source for the ITER neutral beam injector: Project overview and first results of ELISE," IEEE Transactions on Plasma Science, Vol. 42, No.3, (2014).

[12] U. Fantz, et al., "Size scaling of negative hydrogen ion sources for fusion,” AIP conference proceedings 1655, (2015).

[13] M. Fröschle, et al., "Magnetic filter field for ELISE Concepts and Design,” Fusion Eng. Des. 88 (2013) 10151019.

[14] D. Wünderlich, et al., "Optical emission spectroscopy at the large RF driven negative ion test facility ELISE: Instrumental setup and first results," Rev. Sci. Instrum., vol 84, no. 9, pp. 093102-1-093102-6 (2013).

[15] R. Nocentini, et al., "Beam Diagnostic Tools for the negative hydrogen ion source test facility ELISE," Fus. Eng. Des. 88, 913-917 (2013).

[16] R. Nocentini, et al., "Advanced ion beam calorimetry for the test facility ELISE,” AIP Conference Proceedings 1655, (2015). 
[17] F. Bonomo, et al., "BATMAN Beam Properties Characterization by the Beam Emission Spectroscopy Diagnostic," AIP conference proceedings 1655, (2015). 